\title{
INQUÉRITO EPIDEMIOLÓGICO COM HISTOPLASMINA E PARACOCCIDIOIDINA EM ARAPIRACA - ALAGOAS
}

\author{
Marione Cortez Pessoa Santos e Célia Maria Silva Pedrosa
}

\begin{abstract}
Relata-se inquérito utilizando-se antigenos especificos para histoplasmose e paracoccidioidomicose em testes intradérmicos em 107 individuos, com idade de 2 a 71 anos, procedentes do municipio de Arapiraca, Alagoas. Obteve-se $11,21 \%$ de positividade à paracoccidioidina $e$ $14,01 \%$ à histoplasmina. Considerando-se a positividade da paracoccidioidina na população histoplasmina-positiva o resultado demonstra uma porcentagem de 33,33 , enquanto que a positividade da histoplasmina na população paracoccidioidina-positiva foi $41,67 \%$.
\end{abstract}

Palavras-chaves: Paracoccidioidomicose. Histoplasmose. Inquérito epidemiológico. Reacão intradérmica.

A reação intradérmica utilizando antigenos de paracoccidioidina e histoplasmina já foi estudada por vários autores, seja para verificar sua positividade em indivíduos portadores da doença ou, então, na realização de inquéritos epidemiológicos.

No Nordeste do País os relatos são escassos restringindo-se, principalmente, aos Estados de Pernambuco, Bahia e Rio Grande do Norte, segundo referência de Ribeiro ${ }^{8}$ em 1978.

A positividade da reação tem se mostrado significativa como relatam Lacaz, Fava Netto e Raphael, Carandina e Magaldi, Bejgel e Carvalho, Ribeiro e Auto 25678 .

Com o objetivo de trazer subsidios adicionais à epidemiologia da Paracoccidioidomicose e Histoplasmose, onde eles são escassos, foi feito o presente estudo.

\section{MATERIAL E MÉTODOS}

$O$ inquérito foi realizado em população da zona rural do Estado de Alagoas, município de Arapiraca, localidade de Pau D'arcos, situada a $136 \mathrm{~km}$ de Maceió, a região situada no agreste apresenta clima temperado e a agricultura está voltada para o fumo e algodão. $\mathrm{O}$ estudo foi efetuado em 107 pessoas, escolhidas aleatoriamente, sendo $27(25,2 \%)$ do sexo masculino e $80(74,8 \%)$ do feminino; a idade variou entre 2 e 71 anos.

\footnotetext{
* Ambos os antígenos foram produzidos e padronizados na Secção de Sorologia do Departamento de Microbiologia e Imunologia do Instituto de Ciências Biomédicas da Universidade de São Paulo e gentilmente cedidos pelo Prof. Celeste Fava Netto.
}

Departamento de Clinica Médica, Centro de Ciências da Saúde, Universidade Federal de Alagoas, Maceió, AL.

Endereço para correspondencia: Profa Marione Cortez P. Santos. Disc. Doencas Infecciosas e Parasitárias/UFAL. Campus Universitário, 57000 Maceió, AL, Brasil.

Recebido para publicação em 15/04/88.
Os individuos não apresentavam história de doença na época da pesquisa. Todos foram submetidos às provas intradérmicas com paracoccidioidina $e$ histoplasmina. Dados pessoais foram registrados em ficha individual, incluindo tempo de residência, procedência e certos hábitos como mascar folhas ou palitar com gravetos, fumar cigarros de palha, fazer toalete anal com vegetais, contato com animais, inclusive, o morcego.

Nas provas intradérmicas os antígenos* utilizados foram:

1. paracoccidioidina, antígeno polissacáride do Paracoccidioides brasiliensis, na diluição de $1: 10 \mathrm{em}$ solução fisiológica estéril e mertiolatada a 1: 5000 , produzido e padronizado por Fava Netto e Raphael (1961) e

2. histoplasmina, filtrado obtido de única amostra de Histoplasma capsulatum diluído a 1: 1000, nos mesmos parâmetros anteriores.

As provas intradérmicas foram realizadas após assepsia da pele com álcool, injetando-se $0,1 \mathrm{ml} \mathrm{da}$ diluição indicada de cada antígeno, em injeção intradérmica, usando seringas de $1 \mathrm{ml}$, estéreis, com agulhas de bisel curto $(10 \times 4,5)$, do tipo descartável. As aplicações foram feitas, simultaneamente, em um mesmo individuo, por dois operadores, tendo sido padronizado a face anterior do antebraço direito para a paracoccidioidina e o antebraço esquerdo para a histoplasmina. Em todos os casos as leituras foram realizadas após 24 e 48 horas da injeção do antígeno, anotando-se o diâmetro transverso da enduração $e$ eritema locais. Utilizou-se para tais medidas, régua transparente milimetrada. Foram consideradas positivas todas as provas com enduração medindo $5 \mathrm{~mm}$ ou mais de diâmetro.

\section{RESULTADOS}

$\mathrm{Na}$ Tabela 1 verifica-se a distribuição das reações positivas. Dos 107 individuos submetidos às provas intradérmicas, houve uma maior prevalência de positividade à histoplasmina $(14,01 \%)$. Em resposta à paracoccidioidina, 12 pacientes reagiram positivamente $(11,21 \%)$. Nos pacientes paracoccidioidino- 
Santos MCP, Pedrosa CMS. Inquérito epidemiológico com histoplasmina e paracoccidioidina em Arapiraca-Alagoas. Revista da Sociedade Brasileira de Medicina Tropical 23: 213-215, out-dez, 1990.

Tabela 1 -Distribuição das reações intradérmicas positivas à paracoccidioidina e histoplasmina no Municipio de Arapiraca Alagoas, de acordo com a faixa etária.

\begin{tabular}{|c|c|c|c|c|c|c|c|}
\hline \multirow{2}{*}{\multicolumn{2}{|c|}{ Faixa etária }} & & \multicolumn{5}{|c|}{ Provas intradérmicas positivas } \\
\hline & & & \multirow[t]{2}{*}{$\mathrm{N}$} & \multicolumn{2}{|c|}{ Paracoccidioidina } & \multicolumn{2}{|c|}{ Histoplasmina } \\
\hline & & & & $\mathbf{N}$ & $\%$ & $\mathbf{N}$ & $\%$ \\
\hline $\begin{array}{r}0 \\
5 \\
10 \\
15 \\
20 \\
30 \\
40\end{array}$ & $\begin{array}{l}\leftarrow 5 \\
\vdash 10 \\
\leftarrow 15 \\
\vdash 20 \\
\vdash 30 \\
\leftarrow 40 \\
\leftarrow 50 \\
>50\end{array}$ & - & $\begin{array}{r}10 \\
18 \\
14 \\
12 \\
17 \\
16 \\
11 \\
9\end{array}$ & $\begin{array}{l}0 \\
0 \\
0 \\
2 \\
3 \\
2 \\
1 \\
4\end{array}$ & $\begin{array}{r}0,00 \\
0,00 \\
0,00 \\
16,67 \\
25,00 \\
16,67 \\
8,33 \\
33,33\end{array}$ & $\begin{array}{l}1 \\
0 \\
0 \\
1 \\
5 \\
3 \\
3 \\
2\end{array}$ & $\begin{array}{r}6,67 \\
0,00 \\
0,00 \\
6,67 \\
33,33 \\
20,00 \\
20,00 \\
13,33\end{array}$ \\
\hline \multicolumn{2}{|c|}{ Total } & & 107 & 12 & 100,00 & 15 & 100,00 \\
\hline
\end{tabular}

Tabela 2 -Reações intradérmicas positivas à paracoccidioidina. Reações cruzadas.

\begin{tabular}{lcccc}
\hline Paracoccidioidina & & & \multicolumn{2}{c}{ Paracoccidioidina-histoplasmina } \\
\cline { 1 - 2 } $\mathrm{N}$ & $\%$ & $\mathrm{~N}$ & $\%$ \\
\hline 7 & 58,33 & 5 & 41,67 \\
\hline
\end{tabular}

Tabela 3 -Reações intradérmicas positivas à histoplasmina. Reações cruzadas.

\begin{tabular}{lcrrr}
\hline Histoplasmina & \multicolumn{2}{c}{ Histoplasmina-paracoccidioidina } \\
\hline $\mathrm{N}$ & $\%$ & & \multicolumn{2}{c}{$\%$} \\
\cline { 2 - 5 } 10 & 66,67 & 5 & 33,33 \\
\hline
\end{tabular}

positivos 5 reagiram, também, à histoplasmina. No grupo histoplasmino positivo 5 individuos reagiram positivamente à paracoccidioidina.

As Tabelas 2 e 3 apresentam os resultados à histoplasmina e paracoccidioidina considerando-se a positividade de cada prova isoladamente e em conjunto; calculando-se a porcentagem de indivíduos histoplasmino positivos na população paracoccidioidino positiva a partir destes dados, observa-se o índice de positividade de $41,67 \%$; a porcentagem de paracoccidioidino positivos na população histoplasmino positivos foi de $33,33 \%$.

Houve maior positividade para ambas as reações quando a leitura foi realizada após 24 horas. Em relação ao tempo de permanência na região, 91 indivíduos sempre residiram na área enquanto 11 eram procedentes de outras regiōes do Estado de Alagoas e 5 de outros Estados (4 de Pernambuco e 1 de São Paulo). A positividade da paracoccidioidina foi de $7,7 \%$ para individuos que sempre residiram na região, enquanto que a positividade à histoplasmina foi de $12,1 \%$ no mesmo grupo. A maior parte dos individuos apresentava contato com animais, principalmente cães, gatos, morcegos, bovinos, caprinos e suínos, bem como convive com a lavoura, tendo o hábito de mascar gravetos, realizar toalete anal com folhas.

\section{DISCUSSÃO}

No presente inquérito, verifica-se que a infecção ocorreu com maior freqüència nos grupos etários entre 16 e 40 anos, dados concordantes com os de Campos \& Fava Netto ${ }^{3}$ e Ribeiro ${ }^{8}$. Os individuos que mostraram positividade a ambas reações, na sua maior parte, sempre residiram no municipio de Arapiraca e conviviam com animais e referiam uso de folhas como emplastro, inclusive em úlceras de pele, hábito comum no interior do Estado.

A positividade a ambos testes intradérmicos demonstra que a histoplasmose e paracoccidioidimicose - infecção existem em Alagoas; sua importância reside no fato de alertar a classe médica sobre a existência da paracoccidioidomicose e histoplasmose - doença em região onde estas patologias são pouco diagnosticadas, seja nas suas formas subclinicas ou mesmo nas formas clássicas da doença em que outras patologias são diagnosticadas inadvertidamente. 
$O$ fato de encontrarmos reações cruzadas faz elevar o percentual de $11,21 \%$, de reaçóes positivas à paracoccidioidina na população em geral, para $33,33 \%$; do mesmo modo, na população histoplasmino positiva o percentual de $14,01 \%$ encontrado na população em geral, modifica-se para 41,66\% (Tabelas 2 e 3 ).

Tais dados indicam que reaçōes cruzadas ocorrem em ambos os sentidos.

\section{SUMMARY}

Paracoccidioidin and histoplasmin intradermic tests were performed in 107 inhabitants in Arapiraca, Alagoas. The epidemiological survey was performed in both sexes and the ages varied from 2 to 71 years. Positive results with paracoccidioidin test wiere recorded in $11 \%$ and the histoplasmin test was positive in $14 \%$. With the positivity of paracoccidioidin in the histoplasmin positive population the result shows a percentage of $33,33 \%$, while with the positivity of histoplasmin in the paracoccidioidin positive population was $41 \%$.

Key-words: Paracoccidioidomycosis. Histoplasmosis. Skin tests. Epidemiologic survey.

\section{AGRADECIMENTOS}

Agradecemos ao Prof. Celeste Fava Netto que gentilmente nos cedeu os antigenos, bem como pela revisão e sugestões dadas.

\section{REFERÊNCIAS BIBLIOGRÁFICAS}

1. Auto HJF. Inquérito histoplasmínico em Alagoas. Consulta 1: 19-20, 1980.

2. Bejgel I, Carvalho A. Inquérito histoplasmínico em Usina de Acúcar no Estado do Rio de Janeiro. Revista Brasileira de Tuberculose e Doenças Toráxicas 29: 39-49, 1961.

3. Campos CM, Fava Netto C. Reações intradérmicas de paracoccidioidina e de histoplasmina em habitantes urbanos de Bragança Paulista, estado de São Paulo, Brasil. Revista do Instituto de Medicina Tropical de São Paulo 20: 289-292, 1978.

4. Carandina L, Magaldi C. Inquérito sobre blastomicose sul-americana pela intradermo-reação em uma comunidade rural do municipio de Botucatu, SP (Brasil). Revista de Saúde Pública, São Paulo 8: 171-179, 1974.

5. Fava Netto C, Raphael A. A reação intradérmica com polissacáride do Paracoccidioides brasiliensis na blastomicose sul-americana. Revista do Instituto de Medicina Tropical de Sảo Paulo 3: 161-165, 1961.

6. Lacaz CS. Lesões pulmonares na Blastomicose sulamericana: inquérito preliminar com a paracoccidioidina. O Hospital 39: 405-422, 1951.

7. Lacaz CS, Passos Filho MCR, Fava Netto C. Macarron B. Contribuiçāo para o estudo de "Blastomicose infecção". Inquérito com a paracoccidioidina. Estudo sorológico e clínico radiológico dos paracoccidioidinopositivos. Revista do Instituto de Medicina Tropical de São Paulo 1: 245-259, 1959.

8. Ribeiro MAG. Contribuição ao estudo da histoplasmose e paracoccidioidomicose no Estado do Rio Grande do Norte. Tese de Mestrado, Universidade Federal do Rio Grande do Norte, Natal, 1978. 